\title{
Structural Reliability and Its Sensitivity Analysis by Hybrid Markov Chain with Correlated Variables
}

\author{
Ganqing Zhang*, Yanghui Xiang, Lei Guo, Huixin Guo, Yonghong Nie \\ Department of Mechanical \& Electrical Engineering, Changsha University, Changsha, Hunan, China. \\ *Corresponding author. Tel.: +86-13667388819; email: hnyyzgq@163.com \\ Manuscript submitted December 25, 2015; accepted March 8, 2016. \\ doi: 10.17706/jsw.11.5.481-493
}

\begin{abstract}
In the practical engineering, to answer the small failure probabilities with correlated highdimensional variables, the subset simulation (SS) is combined together with the Monte Carlo simulation and importance sampling (IS) method. The samples from the probability density functions of the importance sampling are used to construct the intermediate failure events, by which the small failure probabilities are turned into a hybrid Markov chain, which is a continuous product made of a series large failure probability or conditional failure probability that is easily answered, on which the structural reliability sensitivity can be efficiently simulated by directly obtaining the samples with correlated ones. Finally, the 3 planetary carriers of 3-stages planetary gear reducer in the earth-pressure-balance shield machine are as illustrative examples to check the algorithm above, the results show that the SS of the IS with correlated variables can highly simulate failure probability and its sensitivity.
\end{abstract}

Key words: Hybrid markov chain, reliability, reliability sensitivity, subset simulation, importance sampling, correlated variables.

\section{Introduction}

The correlation between variables distinctly affects the structural reliability and its sensitivity in the real physical structure, therefore, that it is no doubt considering the correlation between them is more practicable when the structural index above is analyzed. Nowadays, the general methods to solve the problems of Reliability Sensitivity (RS) with correlated variables are the transformed method of Monte Carlo[1] and its direct method[2], the ideas of the former are simple, and the most methods to answer the RS are specifically developed according to the independent variables, they are thus favored by multiple scholars. For the latter, however, it is unnecessary to transform the RS, so it is more direct than the former when we answer the RS, what's more, the most current methods to answer the RS with independent variables can be used to analyze the RS with correlated ones once the correlated samples are obtained, while it is also not difficult to produce. The traditional methods, however, are not suitable to be used to solve the impossible problems that are known as high-dimensional small probability [3] in the reliability engineering field due to lack of precision [4] or low efficiency [5]. Subset simulation (SS)[6]-[8] is a highly efficient reliability analysis method, which is specially put forward for small failure probabilities, it divides the original probability space into a series of subsets which are of gradual inclusion relation by introducing reasonable intermediate failure events, and the small failure probability can be represented by a continuous product of the larger conditional failure probabilities (CFP) than itself which are highly 
efficiently answered by numerical simulation method [9]. The document [10] proposes the reliability analysis method based on the SS of Markov Chain Monte Carlo(MCMC), since the pre-production samples from the MCMC are premature and the correlation exists in the conditional samples, which influence its efficiency to certain degree. The document [11] puts forward the RS analysis method based on SS and IS with independent variables, it is high efficient and accurate for it inherits the advantages of SS and IS and avoids the disadvantage of MCMC simulation. The paper, therefore, expands the independent variables to the correlated ones on the basis of above analysis, and the partial derivatives of CFP with respect to the distribution parameters of the basic variables are transformed into the partial ones of CFP with respect to the parameters including the correlation coefficient between them, the stochastic samples with correlated variables are directly obtained by the probability density function (PDF) of the intermediate failure events to efficiently estimate the partial derivatives of CFP with respect to the distribution parameters including the correlation coefficient and directly draw out the computational formulae of RS with correlated variables.

\section{Monte Carlo Numerical Simulation for Solving the RS with Correlated Variables}

In reliability analysis, structural failure probability can be represented by

$$
P_{f}=\int_{F} f_{X}(x) d x
$$

here, $F$ denotes the structural failure region.

Therefore, the RS with correlated variables can written as

$$
\frac{\partial P_{f}}{\partial \theta_{x_{k}}}=\int_{F} \frac{\partial f_{X}(x)}{\partial \theta_{x_{k}}} d x=\int_{R^{n}} \frac{\partial f_{X}(x)}{\partial \theta_{x_{k}}} \frac{I_{F}(x)}{h(x)} h(x) d x=E\left(\frac{\partial f_{X}(x)}{\partial \theta_{x_{k}}} \frac{I_{F}(x)}{h(x)}\right) \quad(i=1,2, \cdots, n)
$$

In Eq.(2), $I_{F}(x)$ is the indicator function of the failure region $F$, when $x \in F, I_{F}(x)=1$, otherwise, $I_{F}(x)=0 . R^{n}$ is $\mathrm{n}$-dimensional variable space. The introduced function $h(x)$ is n-dimensional PDF. In Eq.(2), its mathematical expectation can be resolved by drawing correlated samples from the function $h(x)$.

\section{Reliability Analysis based on HMC SS}

\subsection{Basic Principle of MC SS}

For the most practical engineering problems, their failure probability is very small, and it is difficult to accept computational expense when we directly simulate them, therefore, Au et al.[3] presented a SS method whose basic ideas are that the small failure probability is expressed as a continuous product of the larger CFP by introducing reasonable intermediate failure events shown in Fig.1. If the failure domain of performance function (PF) $g(x)$ is defined as $F=\{x \mid g(x) \leq 0\}$.Assume $b_{1}>b_{2}>\cdots>b_{l}=0$ as a decreasing sequence of the threshold values of failure events $F_{k}=\left\{x \mid g(x) \leq b_{k}\right\}(k=1, \cdots, l)$, then the failure events satisfy the following relations, $F_{1} \supset F_{2} \supset \cdots \supset F_{l}=F$, and $F_{k}=\bigcap_{i=1}^{k} F_{i}(k=1,2, \cdots, l)$. According to the multiplication theorem and the definition of conditional probability in the probability theory, the failure probability can be defined as the following MC

$$
P_{f}=P(F)=P\left(F_{1}\right) \cdot \prod_{i=2}^{l} P\left(F_{i} \mid F_{i-1}\right)
$$

Define $P_{1}=P\left(F_{1}\right), P_{i}=P\left(F_{i} \mid F_{i-1}\right)(i=2,3, \cdots, l)$, the failure probability in Eq.(3) can be rewritten as 


$$
P_{f}=\prod_{i=1}^{l} P_{i}
$$

As can be seen from Eq.(4), obviously, $P_{f} \ll<P_{i}<1$, therefore, for $P_{i}$, it is much easier to answer than $P_{f}$.

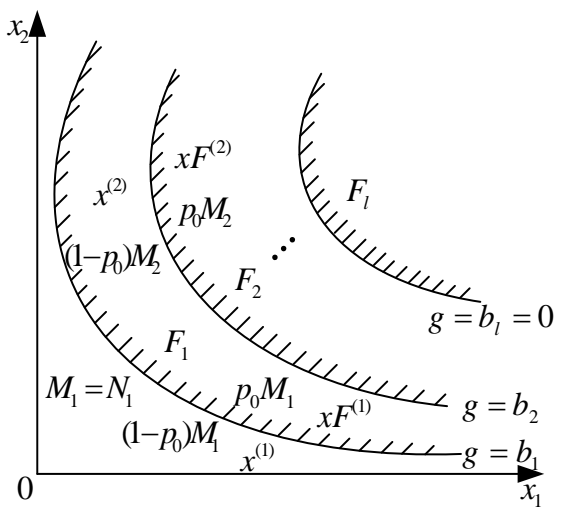

Fig. 1. Schematic diagram of subset simulation.

\subsection{Course of MC SS}

In Eq.(4), $P_{1}$ can be estimated by direct Monte Carlo method, and the conditional failure probability $P_{i}(i=2,3, \cdots, l)$ can be estimated by drawing conditional samples.

$$
\begin{gathered}
\hat{P}_{1}=\frac{1}{N_{1}} \sum_{k=1}^{N_{1}} I_{F_{1}}\left[x_{k}^{(1)}\right] \\
\hat{P}_{i}=\frac{1}{N_{i}} \sum_{k=1}^{N_{i}} I_{F_{i}}\left[x_{k}^{(i)}\right] \quad i=2,3, \cdots, l
\end{gathered}
$$

In Eq.(5) $\sim(6), I_{F_{i}}\left[x_{k}^{(i)}\right](i=1, \cdots, l)$ is an indicator function, when $x_{k}^{(i)} \in F_{i}, I_{F_{i}}\left[x_{k}^{(i)}\right]=1$, otherwise, $I_{F_{i}}\left[x_{k}^{(i)}\right]=0$. Here, $x_{k}^{(1)}$ is the $k$ th sample of the independent and identical distribution $N_{1}$ samples drawn from the combined PDF $f_{X}(x)$, and $x_{k}^{(i)}(i=2,3, \cdots, l)$ is the $k$ th sample of the independent and identical distribution $N_{i}$ samples drawn from the conditional PDF $q\left(x \mid F_{i-1}\right)=I_{F_{i-1}}[x] f_{X}(x) / P\left(F_{i-1}\right)$. Although the direct MCS can be applied to obtain conditional samples of the conditional PDF $q\left(x \mid F_{i-1}\right)$, it is not efficient since, on an average, it should take $1 / \prod_{j=1}^{i-1} P_{j}$ samples before one conditional sample occurs. What's more, in general, the task of efficiently simulating conditional samples is not trivial[12].

For the above joint PDF $f_{X}(x)$, when the n-dimensional variables $x=\left(x_{1}, x_{2}, \cdots, x_{n}\right)^{T}$ are normal distribution, and there exists the correlation between variables, it may be negligible among three or more variables, it can be expressed as

$$
f_{X}(x)=(2 \pi)^{-\frac{n}{2}}\left|C_{x}\right|^{-\frac{1}{2}} \exp \left[-\frac{1}{2}\left(x-\mu_{x}\right)^{T} C_{x}^{-1}\left(x-\mu_{x}\right)\right]
$$

where

$$
C_{x}=\left[\begin{array}{cccc}
\sigma_{x_{1}}^{2} & \rho_{x_{1} x_{2}} \sigma_{x_{1}} \sigma_{x_{2}} & \cdots & \rho_{x_{1} x_{n}} \sigma_{x_{1}} \sigma_{x_{n}} \\
\rho_{x_{2} x_{1}} \sigma_{x_{1}} \sigma_{x_{2}} & \sigma_{x_{2}}^{2} & \cdots & \rho_{x_{2} x_{n}} \sigma_{x_{2}} \sigma_{x_{n}} \\
\vdots & \vdots & & \vdots \\
\rho_{x_{n} x_{1}} \sigma_{x_{1}} \sigma_{x_{n}} & \rho_{x_{n} x_{2}} \sigma_{x_{2}} \sigma_{x_{n}} & \cdots & \sigma_{x_{n}}^{2}
\end{array}\right]
$$


Eq. (8) is the covariance matrix of the variables $x, C_{x}^{-1}$ is their inverse matrix, $\left|C_{x}\right|$ is the determinant of matrix $C_{x}, \mu_{x}=\left(\mu_{x_{1}}, \mu_{x_{2}}, \cdots, \mu_{x_{n}}\right)^{T}$ is the mean vector of the variables $x$. Here, $\mu_{x_{i}}$ and $\sigma_{x_{i}}$ is their mean and mean square error, respectively, $\rho_{x_{i} x_{j}}$ is the correlation coefficient of the variable $x_{i}$ and $x_{j}$.

\subsection{Reliability Analysis Based on HMC SS}

\subsubsection{Definition and analysis idea of reliability}

In the course of using MC SS to analyze the failure probability, the initial failure probability $\hat{P}_{1}$ is firstly simulated by direct Monte Carlo, then the conditional samples are simulated by the IS method, namely, the PDF of the IS method in the each subset is constructed step by step, and we can obtain the PDF of the LSE, finally reach the estimate value $\hat{P}_{i}(i=2,3, \cdots, l)$ of each CFP, so the Eq.(3) of the continuous product constitutes a HMC, namely, the Monte Carlo simulation hybridize with IS method. Since the method does not need to select the proposal distribution of MC, the correlation between conditional samples can be avoided to some certain, which increase the efficiency of drawing samples and the robustness of the method.

When analyzing the reliability by the SS and IS, the CFP is calculated by the following formula.

$$
P_{i}=\int \cdots \int_{F_{i}} I_{F_{i}}(x) q\left(x \mid F_{i-1}\right) d x=\int \cdots \int_{\Omega} \frac{I_{F_{i}}(x) q\left(x \mid F_{i-1}\right)}{h_{i-1}(x)} h_{i-1}(x) d x=E\left[\frac{I_{F_{i}}(x) q\left(x \mid F_{i-1}\right)}{h_{i-1}(x)}\right]
$$

If the mathematical expectation $E[\cdot]$ is estimated by the samples mean, then the estimated values of the CFP are

$$
\hat{P}_{i}=\frac{1}{N_{i}} \sum_{k=1}^{N_{i}} \frac{I_{F_{i}}\left(x_{k}^{(i)}\right) q_{X}\left(x_{k}^{(i)} \mid F_{i-1}\right)}{h_{i-1}\left(x_{k}^{(i)}\right)}=\frac{1}{N_{i}} \sum_{k=1}^{N_{i}} I_{F_{i}}\left(x_{k}^{(i)}\right) \frac{f_{X}\left(x_{k}^{(i)}\right)}{h_{i-1}\left(x_{k}^{(i)}\right)} \frac{1}{P\left(F_{i-1}\right)} \quad(i=2, \cdots, l)
$$

\subsubsection{Implementation mechanism}

During the course of HMC SS, it is critical that how to select the intermediate failure events $\left\{F_{1}, F_{2}, \cdots, F_{l}\right\}$ that are of containment relationship step by step to reduce the total samples. For the levels $l$ and the sampling points $N_{i}$ of every level, if one decreases, the other will necessarily increases, therefore, the following compromise between them is proposed when the structural reliability is analyzed in the whole region, namely, the conditional probability $p_{0}$ (e.g. $p_{0}=1 \times 10^{-1}$ ) is preset beforehand, the number of the level is adaptively selected according to the practicable need. The concrete courses are as follows which are described in Fig.1 to easily understand and operate them.

1) In the feasible region, $N_{1}$ samples $\left\{x_{k}^{(1)}: k=1,2, \cdots, N_{1}\right\}$ are produced by direct Monte Carlo simulation who obey joint $\operatorname{PDF} f_{X}(x)$, and who are independent and correlated between variables.

2) We can get the corresponding response values $\left\{g\left(x_{k}^{(1)}\right): k=1,2, \cdots, N_{1}\right\}$ after all samples are substituted into the PF $g(x)$, and they are sorted in the decreasing order. Define $M_{1}=N_{1}$, and the $\left[\left(1-p_{0}\right) M_{1}\right]$ th response value is the threshold $b_{1}$ of the intermediate failure event $F_{1}=\left\{x \mid g(x) \leq b_{1}\right\}$, namely, $b_{1}=g\left(x_{\left[\left(1-p_{0}\right) M_{1}\right]}^{(1)}\right)$, the failure probability $P_{1}=P\left(F_{1}\right)$ can thus be estimated by $\hat{P}_{1}=p_{0}$.

3) After the $p_{0} M_{i-1}$ samples are stored in $x F(i-1)$ who fall in the failure region $F_{i-1}(i=2,3, \cdots, l)$, the point that maximizes the original joint PDF value is singled out of them, and it is as the mean to construct the IS PDF $h_{i-1}(x)$.In the feasible region, the $N_{i}$ samples are produced who obey PDF 
$h_{i-1}(x)$.Among them, there are $M_{i}$ samples that fall in the failure region $F_{i-1}(i=2,3, \cdots, l)$, and their distribution obeys conditional PDF $h_{i-1}\left(x \mid F_{i-1}\right)$.

4) We can get the corresponding response values $\left\{g\left(x_{k}^{(1)}\right): k=1,2, \cdots, N_{1}\right\}$ after all above samples are substituted into the PF $g(x)$, and they are sorted in the decreasing order, and the $\left[\left(1-p_{0}\right) M_{i}\right]$ th response value is the threshold $b_{i}$ of the intermediate failure event $F_{i}=\left\{x \mid g(x) \leq b_{i}\right\}$, namely, $b_{i}=g\left(x_{\left[\left(1-p_{0}\right) M_{i}\right]}^{(i)}\right)$, then the estimated value $\hat{P}\left(F_{i} \mid F_{i-1}\right)$ of the CFP is computed according to Eq.(10), here, $\hat{P}\left(F_{i-1}\right)=\prod_{j=1}^{i-1} \hat{P}_{j}$.

5) Repeat steps (3) $\sim(4)$ until the response value of the $\left[\left(1-P_{0}\right) M_{i}\right]$ th sample $g\left(x_{\left[\left(1-P_{0}\right) M_{i}\right]}^{(i)}\right)<0$. After the position is found that is closest to 0 and $\geq 0$, suppose $l=i, b_{l}=0, F=F_{l}$, the estimated value $\hat{P}\left(F_{l} \mid F_{l-1}\right)$ is still computed according to Eq.(10).

6) Compute the estimated value of the structural failure probability $\hat{P}_{f}=\prod_{i=1}^{l} \hat{P}_{i}$.

As can be seen from the above process, the SS based on HMC is not subject to variable dimensions and the nonlinear degree of LSE, so it is very suitable for the highly nonlinear small failure probabilities.

\section{RS Analysis Based on HMC SS}

The RS refers to the partial derivatives of failure probability with respect to the distribution parameters of the basic variables. The basic ideas of the RS based on HMC SS are that the sensitivity of the failure probability $P_{f}$ is transformed into the sensitivity form of a series of CFP by using the method that $P_{f}$ is expressed as the continuous product of a series of CFP through HMC SS, and then it is estimated by the conditional samples.

According to Eq.(4), the partial derivatives of failure probability $P_{f}$ with respect to each design variable $\theta_{x_{k}}$ concluding the mean, variance and coefficient of variation are defined by

$$
\begin{gathered}
\frac{\partial P_{f}}{\partial \theta_{x_{k}}}=\sum_{i=1}^{l} \frac{P_{f}}{P_{i}} \frac{\partial P_{i}}{\partial \theta_{x_{k}}} \\
\because P_{1}=\int \cdots \int_{F_{i}} f_{X}(x) d x, \quad P_{i}=\int \cdots \int_{F_{i}} q\left(x \mid F_{i-1}\right) d x(i=2, \cdots, l) \\
\therefore \frac{\partial P_{f}}{\partial \theta_{x_{k}}}=\frac{P_{f}}{P_{1}} \int \cdots \int_{F_{1}} \frac{\partial f_{X}(x)}{\partial \theta_{x_{k}}} d x+\sum_{i=2}^{l} \frac{P_{f}}{P_{i}} \int \cdots \int_{F_{i}} \frac{\partial q\left(x \mid F_{i-1}\right)}{\partial \theta_{x_{k}}} d x \\
=\frac{P_{f}}{P_{1}} \int \cdots \int_{\Omega} \frac{I_{F_{1}}[x]}{f_{X}(x)} \frac{\partial f_{X}(x)}{\partial \theta_{x_{k}}} f_{X}(x) d x+\sum_{i=2}^{l} \frac{P_{f}}{P_{i}} \int \cdots \int_{\Omega} \frac{I_{F_{i}}[x]}{h_{i-1}(x)} \frac{\partial q\left(x \mid F_{i-1}\right)}{\partial \theta_{x_{k}}} h_{i-1}(x) d x
\end{gathered}
$$

Here, $\Omega$ denotes the whole variable space, the expectation of Eq.(12) is

$$
\frac{\partial P_{f}}{\partial \theta_{x_{k}}}=\frac{P_{f}}{P_{1}} \frac{\partial P_{1}}{\partial \theta_{x_{k}}}+\sum_{i=2}^{l} \frac{P_{f}}{P_{i}} \frac{\partial P_{i}}{\partial \theta_{x_{k}}}=\frac{P_{f}}{P_{1}} E\left[\frac{I_{F_{1}}[x]}{f_{X}(x)} \frac{\partial f_{X}(x)}{\partial \theta_{x_{k}}}\right]+\sum_{i=2}^{l} \frac{P_{f}}{P_{i}} E\left[\frac{I_{F_{i}}[x]}{h_{i-1}(x)} \frac{\partial q\left(x \mid F_{i-1}\right)}{\partial \theta_{x_{k}}}\right]
$$

According to the definition of the mathematical expectation, the estimated value of each monomial in Eq.(13) is 


$$
\begin{gathered}
\frac{\partial P_{1}}{\partial \theta_{x_{k}}}=\frac{1}{N_{1}} \sum_{k=1}^{N_{1}} \frac{I_{F_{1}}\left[x_{k}^{(1)}\right]}{f_{X}\left(x_{k}^{(1)}\right)} \frac{\partial f_{X}\left(x_{k}^{(1)}\right)}{\partial \theta_{x_{k}}} \\
\frac{\partial P_{i}}{\partial \theta_{x_{k}}}=\frac{1}{N_{i}} \sum_{k=1}^{N_{i}} \frac{I_{F_{i}}\left[x_{k}^{(i)}\right]}{h_{i-1}\left(x_{k}^{(i)}\right)} \frac{\partial q\left(x_{k}^{(i)} \mid F_{i-1}\right)}{\partial \theta_{x_{k}}}
\end{gathered}
$$

where, the conditional probability of the conditional samples $x_{k}^{(i)}(i=2,3, \cdots, l)$ is

$$
q\left(x_{k}^{(i)} \mid F_{i-1}\right)=\frac{I_{F_{i-1}}\left[x_{k}^{(i)}\right] f_{X}\left(x_{k}^{(i)}\right)}{P\left(F_{i-1}\right)} \quad(i=2,3, \cdots, l)
$$

The partial derivatives of Eq.(16) with respect to $\theta_{x_{k}}$ is

$$
\frac{\partial q\left(x_{k}^{(i)} \mid F_{i-1}\right)}{\partial \theta_{x_{k}}}=\frac{I_{F_{i-1}}\left[x_{k}^{(i)}\right]\left[\frac{\partial f_{X}\left(x_{k}^{(i)}\right)}{\partial \theta_{x_{k}}} P\left(F_{i-1}\right)-f_{X}\left(x_{k}^{(i)}\right) \frac{\partial P\left(F_{i-1}\right)}{\partial \theta_{x_{k}}}\right]}{\left[P\left(F_{i-1}\right)\right]^{2}}(i=2,3, \cdots, l)
$$

The following formula are drawn after Eq.(16) (17) are substituted into Eq.(15)

$$
\begin{aligned}
& \frac{\partial P_{i}}{\partial \theta_{x_{k}}}=\frac{1}{N_{i}} \times \sum_{k=1}^{N_{i}}\left[\frac{I_{F_{i}}\left[x_{k}^{(i)}\right] I_{F_{i-1}}\left[x_{k}^{(i)}\right]}{h_{i-1}\left(x_{k}^{(i)}\right)} \frac{\left(\frac{\partial f_{X}\left(x_{k}^{(i)}\right)}{\partial \theta_{x_{k}}} P\left(F_{i-1}\right)-f_{X}\left(x_{k}^{(i)}\right) \frac{\partial\left(F_{i-1}\right)}{\partial \theta_{x_{k}}}\right]}{\left[P\left(F_{i-1}\right)\right]^{2}}\right] \\
& \frac{\partial P_{i}}{\partial \theta_{x_{k}}}=\frac{1}{P\left(F_{i-1}\right)} \frac{1}{N_{i}} \times \sum_{k=1}^{N_{i}}\left\{\frac{I_{F_{i}}\left[x_{k}^{(i)}\right]}{h_{i-1}\left(x_{k}^{(i)}\right)}\left[\frac{\partial f_{X}\left(x_{k}^{(i)}\right)}{\partial \theta_{x_{k}}}-\frac{f_{X}\left(x_{k}^{(i)}\right)}{P\left(F_{i-1}\right)} \frac{\partial P\left(F_{i-1}\right)}{\partial \theta_{x_{k}}}\right]\right\} \\
& \frac{\partial P_{i}}{\partial \theta_{x_{k}}}=\frac{1}{P\left(F_{i-1}\right)} \frac{1}{N_{i}} \times \sum_{k=1}^{N_{i}}\left\{\frac{\left.I_{F_{i}}\left[x_{k}^{(i)}\right]\left[\frac{\partial f_{X}\left(x_{k}^{(i)}\right)}{h_{i-1}\left(x_{k}^{(i)}\right)}-\frac{f_{X}\left(x_{k}^{(i)}\right)}{P\left(F_{i-1}\right)} \frac{\partial P\left(F_{i-1}\right)}{\partial \theta_{x_{k}}}\right]\right\}}{}=\frac{1}{P\left(F_{i-1}\right)} \frac{1}{N_{i}} \times \sum_{k=1}^{N_{i}}\left\{\frac{I_{F_{i}}\left[x_{k}^{(i)}\right]}{h_{i-1}\left(x_{k}^{(i)}\right)}\left[\frac{\partial f_{X}\left(x_{k}^{(i)}\right)}{\partial \theta_{x_{k}}}-\frac{f_{X}\left(x_{k}^{(i)}\right)}{P\left(F_{i-1}\right)} \sum_{j=1}^{i-1} \frac{P\left(F_{i-1}\right)}{P_{j}} \frac{\partial P_{j}}{\partial \theta_{x_{k}}}\right]\right\}\right. \\
& =\frac{1}{P\left(F_{i-1}\right)} \frac{1}{N_{i}} \times \sum_{k=1}^{N_{i}}\left\{\frac{I_{F_{i}}\left[x_{k}^{(i)}\right]}{h_{i-1}\left(x_{k}^{(i)}\right)}\left[\frac{\partial f_{X}\left(x_{k}^{(i)}\right)}{\partial \theta_{x_{k}}}-\sum_{j=1}^{i-1} \frac{f_{X}\left(x_{k}^{(i)}\right)}{P_{j}} \frac{\partial P_{j}}{\partial \theta_{x_{k}}}\right]\right\}
\end{aligned}
$$

Eq.(18) is recurrence formula for $P_{j}$ and $\partial P_{j} / \partial \theta_{x_{k}}(j=1, \cdots, i-1)$ are the conditional probability and its sensitivity, respectively, so we can answer $\partial P_{i} / \partial \theta_{x_{k}}$ by using the correlation information of the foregoing $i-1$ monomials. If the PDF $f_{X}(x)$ is the normal distribution of Eq.(7), the analytical answers of $\partial f_{X}\left(x_{k}^{(i)}\right) / \partial \theta_{x_{k}}$ whose $\theta_{x_{k}}$ is $\mu_{x_{i}}, \sigma_{x_{i}}$ and $\rho_{x_{i} x_{j}}$ are as follows, respectively.

$$
\begin{gathered}
\frac{\partial f_{X}\left(x_{k}^{(i)}\right)}{\partial \mu_{x_{k}}}=f_{X}\left(x_{k}^{(i)}\right)\left[(0, \cdots, 1, \cdots, 0) C_{x}^{-1}\left(x-\mu_{x}\right)\right] \\
\frac{\partial f_{X}\left(x_{k}^{(i)}\right)}{\partial \sigma_{x_{k}}}=f_{X}\left(x_{k}^{(i)}\right) \times\left\{-\frac{1}{2}\left[\left(x-\mu_{x}\right)^{T} \frac{\partial C_{x}^{-1}}{\partial \sigma_{x_{k}}}\left(x-\mu_{x}\right)+\frac{1}{\left|C_{x}\right|} \frac{\partial\left|C_{x}\right|}{\partial \sigma_{x_{k}}}\right]\right\}
\end{gathered}
$$




$$
\frac{\partial f_{X}\left(x_{k}^{(i)}\right)}{\partial \rho_{x_{i} x_{j}}}=f_{X}\left(x_{k}^{(i)}\right) \times\left\{-\frac{1}{2}\left[\left(x-\mu_{x}\right)^{T} \frac{\partial C_{x}^{-1}}{\partial \rho_{x_{i} x_{j}}}\left(x-\mu_{x}\right)+\frac{1}{\left|C_{x}\right|} \frac{\partial\left|C_{x}\right|}{\partial \rho_{x_{i} x_{j}}}\right]\right\}
$$

In essence, the above analysis process follows the following hypothesis that the IS PDF of the probability sensitivity analysis is identical to that of the failure probability. However, there exists a theoretical difference between them, because the points that contribute to maximizing the failure probability do not always fully coincide with those points that do the probability sensitivity. But the difference is negligible when roughly analyzing them, namely, the IS PDF who estimates the failure probability is applied to estimate the probability sensitivity to achieve the high efficiency of drawing samples.

\section{Illustrative Examples}

For the 3-stage planetary gear reducer (whose type is $2 \mathrm{~K}-\mathrm{H}$ ) of some earth-pressure-balance shield machine, whose input torque $T_{s}$ is $1489 \mathrm{~N} . \mathrm{m}$. The material of the carriers is alloy cast steel ZG42GrMo, whose yield strength $\sigma_{s}$ is $400 \mathrm{MPa}$. The basic variables involved in design course are shown in Table 1 and Table 2, the correlated coefficient between variables is set to 0.7 . The carriers are as examples to check the precision and efficiency of the proposed method.

Table 1. Parameter Values of Reducer in Each Stage

\begin{tabular}{llll}
\hline Name & $1^{\text {st }}$ stage & $2^{\text {nd }}$ tage & $3^{\text {rd }}$ stage \\
\hline Teeth number of sun gear $z_{s}$ & 21 & 25 & 24 \\
Teeth number of planetary gear $z_{p}$ & 17 & 19 & 26 \\
Teeth number of inner gear $z_{r}$ & 55 & 63 & 76 \\
planetary gear number $n_{p}$ & 4 & 4 & 5 \\
Modulus $m / \mathrm{mm}$ & 4 & 4 & 5 \\
\hline
\end{tabular}

\subsection{Assumed Conditions}

The resultant force $F$ of both the normal force $F_{n 1}$ between planetary gear and inner gear and the normal force $F_{n 2}$ between it and sun gear acts on its base circle, which causes the stresses in its base circle to generally distribute in semi-cosine formation [13]. Because force $F$ eventually acts on the planet carrier by the axle of the planetary gear, and the connection between planetary gear and its carrier is locked by interference fit and positioning bolt, the types of the force that acts on the carrier (e.g. alternation and impact) are similar to those of the force that acts on the base circle of the planetary gear. To eliminate the adverse effects on the carrier resulting from the additional stresses and to simplify the analysis process, the following supposed conditions are proposed. 1) The distribution form of the stresses around the axle hole of planet carrier is also semi-cosine. 2) The planetary axle is rigid, that is the axle isn't deformed by force, by which the additional stresses aren't caused around the axle hole. 3) The impact of unbalanced load isn't considered. Namely, it is considered that the force which acts on the bilateral walls is always equal, and their thicknesses are also same. 4) The distribution forms of all variables are normal.

\subsection{Basic Variables}

For any axle hole of the planetary gears in the carrier, the stress depends entirely on the arc area when the force that acts on the carrier is invariant, while it is only relevant to the radius of the planetary axle $R$ and the thickness of the carrier $B$. The basic variables, thereby, are $x=\left[x_{1}, x_{2}\right]^{T}=[R, B]^{T}$. 
Table 2. Parameters of Random Variables and the Safety Coefficient in Every Stage

\begin{tabular}{lllll}
\hline & \multicolumn{3}{l}{ Basic variables $x$} \\
\cline { 2 - 4 } & Name & $\mu_{x} / \mathrm{mm}$ & \multicolumn{1}{l}{$S$} \\
\hline \multirow{2}{*}{ 1st $^{\text {stage }}$} & $R$ & 14 & 0.12 & \multirow{2}{*}{5.5} \\
& $B$ & 18.5 & 0.12 & \\
\hline \multirow{2}{*}{ 2 $^{\text {nd }}$ stage } & $R$ & 19.5 & 0.12 & \multirow{2}{*}{4} \\
& $B$ & 28.5 & 0.12 & \\
\hline \multirow{2}{*}{ 3 $^{\text {rd }}$ stage } & $R$ & 32 & 0.12 & \multirow{2}{*}{3} \\
& $B$ & 28 & 0.12 &
\end{tabular}

Note: The domain of safety coefficient is taken as [2], [6]

\subsection{Mechanical Models}

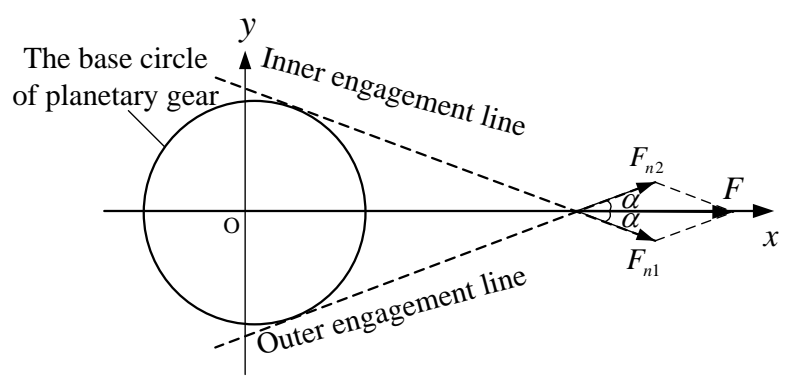

Fig. 2. Force figure of the planetary gear.

According to the theory of gear engagement, both of the normal force $F_{n 1}$ between planetary gear and inner gear and the normal force $F_{n 2}$ between it and sun gear are tangential to its base circle, whose resultant force passes by the centre of the planetary gear and is perpendicular to the centerline $y$-axis. The force that acts on the planetary gear is shown in Fig. 2.

As can be seen from Fig. 2.

$$
F=F_{n 1} \cos \alpha+F_{n 2} \cos \alpha=\frac{F_{t 1}}{\cos \alpha} \cos \alpha+\frac{F_{t 2}}{\cos \alpha} \cos \alpha=F_{t 1}+F_{t 2}
$$

Here, $F_{n 1}$ and $F_{n 2}$ are the normal force between planetary gear and inner gear and the normal force between it and sun gear, respectively. $F_{t 1}$ and $F_{t 2}$ are the tangential force between them, respectively, whose computational formula are referred in the document[14], $\alpha$ is the pressure angle of gear pitch circle, generally, $\alpha=20^{\circ}$.

For the planetary reducer whose type is $2 \mathrm{~K}-\mathrm{H}$, the relation between the torque $T_{s_{i}}(i=1,2,3)$ in every stage is [15].

$$
T_{s_{i}}=\left(1+p_{i-1}\right) T_{s_{i-1}}(i=2,3)
$$

Here, $p$ is referenced in above section.

Suppose the stresses are $\sigma$ in the point $A$ of $\mathrm{x}$-axis, as are shown in Fig.5. Since the distribution form of the stresses around the axle hole of the planet carrier is semi-cosine, in any position with the $x$-axis into $\theta \operatorname{corner}$, its stresses are $\sigma \cos \theta$. After they are decomposed along $\mathrm{x}$-axis and $\mathrm{y}$-axis, the stresses are $\sigma \cos \theta \cdot \cos \theta=\sigma \cos ^{2} \theta$ in the direction of x-axis, and they are $\sigma \cos \theta \cdot \sin \theta$ in the direction of y-axis. If they are integrated in the interval $[-\pi / 2, \pi / 2]$, the force is calculated out in the direction of $y$-axis, in the 
direction of $\mathrm{x}$-axis, however, the resultant force is $B R \int_{-\frac{\pi}{2}}^{\frac{\pi}{2}} \sigma \cos ^{2} \theta d \theta$, which should be equal to $F / 2$ (as the force $F$ acts on the bilateral walls whose force is equal), then

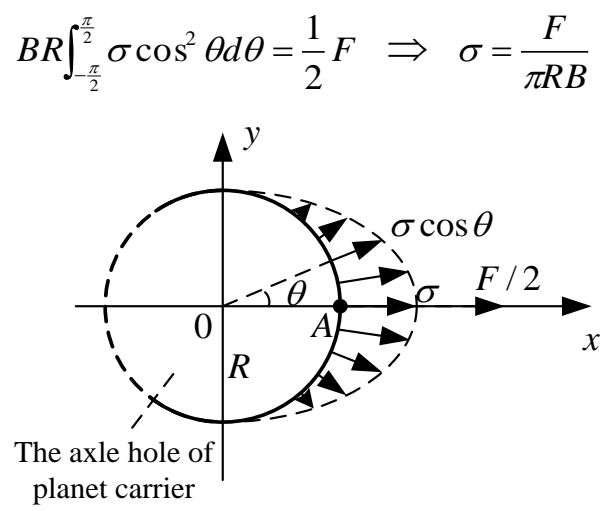

Fig. 3. Stress distribution around the axle hole of the planet carrier.

For the entire carrier, as can be seen from Fig.3, the stress of point $A$ is maximal, so the point $A$ is just the most dangerous point. For point $A$, when fully considering the safety margin, according to the stress-strength interference theory, the PF whose stress is in the form of LSE is represented by

$$
g(x)=\frac{r}{S}-\sigma=\frac{r}{S}-\frac{F}{\pi R B}
$$

where, $x=[R, B]^{T} . r, S, F, R$ and $B$ refer to the allowable stress of material, safety coefficient, the force of planetary gear, the radius of the planetary axle and the thickness of the carrier unilateral wall, respectively.

\subsection{Results Analysis}

The results and sample size that analyze the carriers with the present method and MCS, respectively, are summarized in Table 3. As known from it, the efficiency of the proposed method is higher than that of the MCS.

To confirm the stratification, as described in Fig.1, which the subsets simulate the failure probability, for the failure simulation of the $1 \sim 3$ carriers, the samples that simulate every failure subset are clearly shown in Fig.4 $\sim$ Fig.6, which can perfectly prove that the proposed method does simulate the failure probability by resorting to stratification subsets which are formed by the samples from Monte Carlo and the IS method.

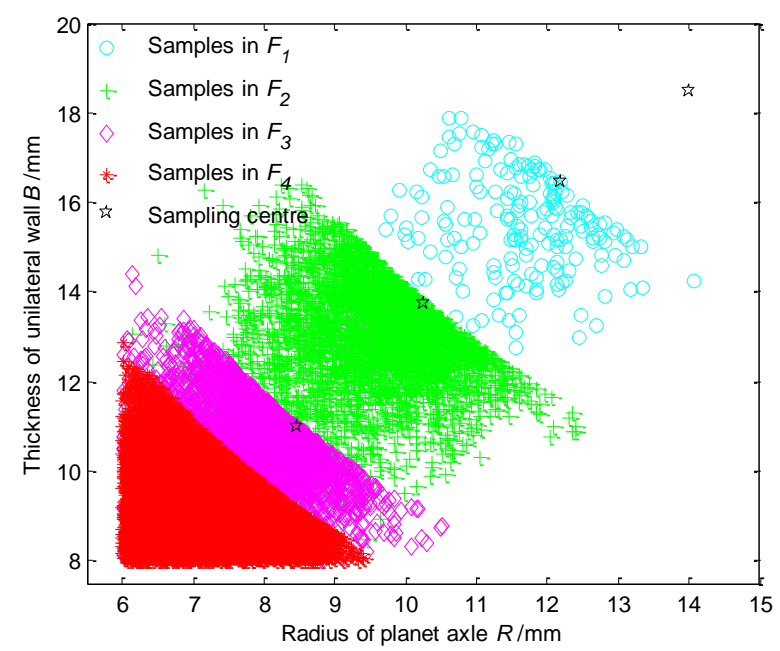

Fig. 4. Simulation of failure subset and sampling centre for the $1^{\text {st }}$ carrier. 


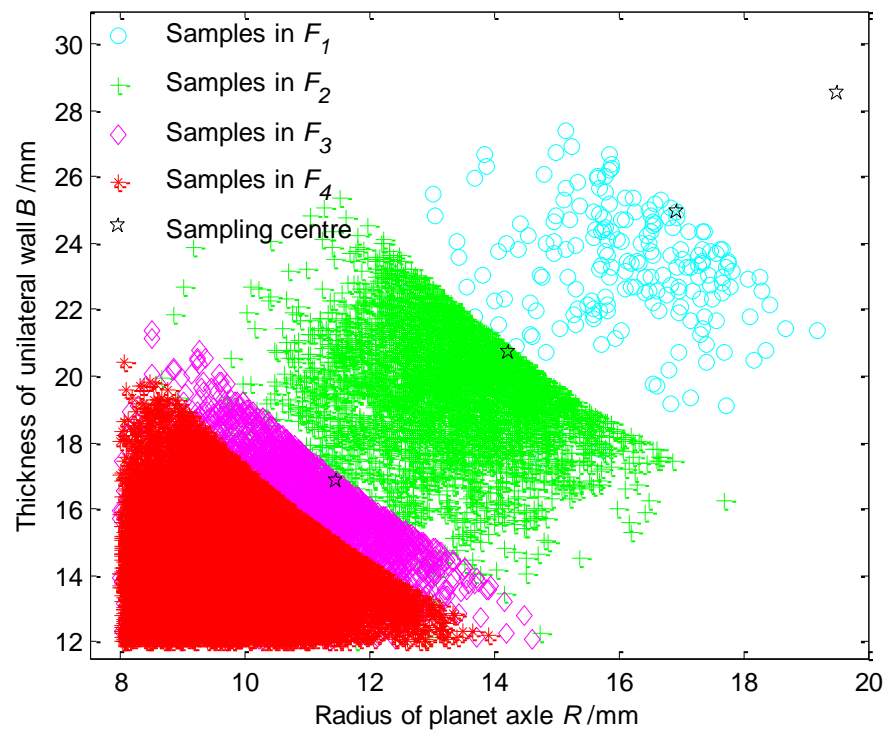

Fig. 5. Simulation of failure subset and sampling centre for the $2^{\text {nd }}$ carrier.

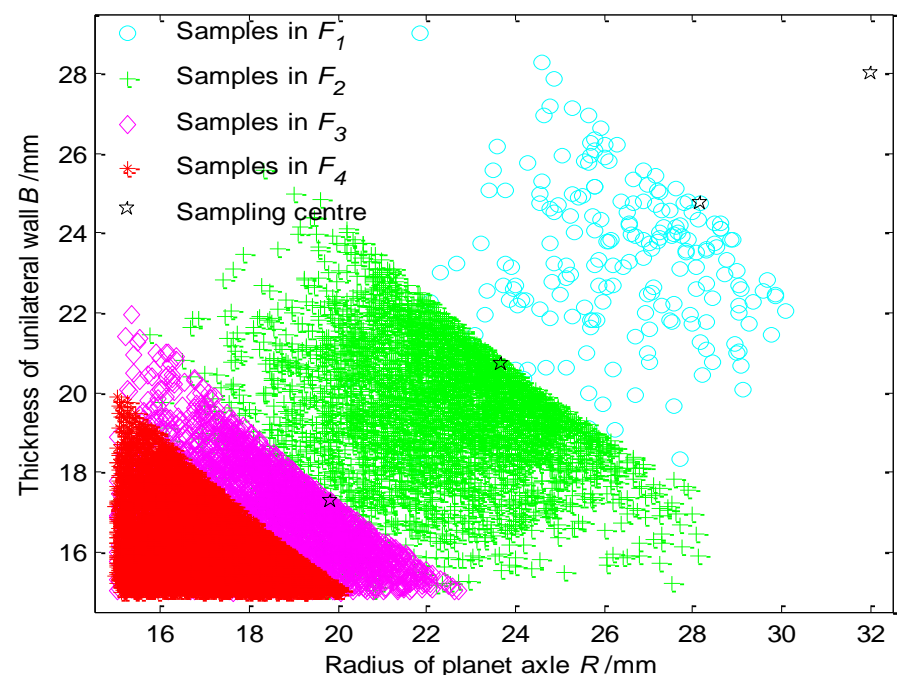

Fig. 6. Simulation of failure subset and sampling centre for the $3^{\text {rd }}$ carrier.

Table 3. Structural Failure Probability and Sample Size Contrast of 3-Stages Carriers Analyzed with MCS and Present Method, Respectively

\begin{tabular}{ccccccc}
\hline \multirow{2}{*}{ Name } & \multicolumn{2}{c}{$1^{\text {st }}$ stage } & \multicolumn{2}{c}{$2^{\text {nd }}$ stage } & \multicolumn{2}{c}{$3^{\text {rd }}$ stage } \\
\cline { 2 - 7 } & $P_{\mathrm{f}}$ & sample size & $P_{\mathrm{f}}$ & sample size & $P_{\mathrm{f}}$ & sample size \\
\hline MCS & $3.2243 \times 10^{-5}$ & $7 \times 10^{7}$ & $5.2529 \times 10^{-5}$ & $7 \times 10^{7}$ & $6.6486 \times 10^{-5}$ & $7 \times 10^{7}$ \\
Present method & $3.0976 \times 10^{-5}$ & $9 \times 10^{4}$ & $5.4695 \times 10^{-5}$ & $9 \times 10^{4}$ & $6.7023 \times 10^{-5}$ & $9 \times 10^{4}$ \\
relative error & $3.93 \%$ & - & $4.12 \%$ & - & $0.81 \%$ & - \\
\hline
\end{tabular}

For the every optimal design point of the $1 \sim 3$ carriers, after the RS of the corresponding structure is analyzed with the present method and MCS, respectively, the results are summarized in Table 4. As can be seen from it, the proposed algorithm can highly simulate the structural RS.

For the every optimal design point of the $1 \sim 3$ carriers, after the RS of the corresponding structure is analyzed with the present method and MCS, respectively, the results are summarized in Table 4. As can be seen from it, the proposed algorithm can highly simulate the structural RS. 
Table 4. Structural RS Contrast of 3-Stages Carriers Analyzed with MCS and Present Method, Respectively

\begin{tabular}{|c|c|c|c|c|c|c|c|c|c|}
\hline & & $1^{\text {st }}$ stage & & & $2^{\text {nd }}$ stage & & & $3^{\text {rd }}$ stage & \\
\hline Name & MCS & $\begin{array}{l}\text { present } \\
\text { method }\end{array}$ & $\begin{array}{c}\text { relative } \\
\text { error }\end{array}$ & MCS & $\begin{array}{l}\text { present } \\
\text { method }\end{array}$ & $\begin{array}{c}\text { relative } \\
\text { error }\end{array}$ & MCS & $\begin{array}{l}\text { present } \\
\text { method }\end{array}$ & $\begin{array}{c}\text { relative } \\
\text { error }\end{array}$ \\
\hline$\partial P_{f} / \partial \mu_{R}$ & $\begin{array}{c}-4.5512 \\
\times 10^{-5}\end{array}$ & $\begin{array}{c}-4.4346 \\
\times 10^{-5}\end{array}$ & $2.56 \%$ & $\begin{array}{c}-5.0681 \\
\times 10^{-5}\end{array}$ & $\begin{array}{c}-4.9484 \\
\times 10^{-5}\end{array}$ & $2.36 \%$ & $\begin{array}{c}-4.9760 \\
\times 10^{-5}\end{array}$ & $\begin{array}{c}-4.8870 \\
\times 10^{-5}\end{array}$ & $1.79 \%$ \\
\hline$\partial P_{f} / \partial \mu_{B}$ & $\begin{array}{c}-3.2928 \\
\times 10^{-5}\end{array}$ & $\begin{array}{c}-3.2399 \\
\times 10^{-5}\end{array}$ & $1.61 \%$ & $\begin{array}{c}-3.5354 \\
\times 10^{-5}\end{array}$ & $\begin{array}{c}-3.5456 \\
\times 10^{-5}\end{array}$ & $0.28 \%$ & $\begin{array}{c}-2.7898 \\
\times 10^{-5}\end{array}$ & $\begin{array}{c}-2.9552 \\
\times 10^{-5}\end{array}$ & $5.93 \%$ \\
\hline$\partial P_{f} / \partial \mu$ & $\begin{array}{c}5.6175 \\
\times 10^{-5}\end{array}$ & $\begin{array}{c}5.4923 \\
\times 10^{-5}\end{array}$ & $2.23 \%$ & $\begin{array}{c}6.1794 \\
\times 10^{-5}\end{array}$ & $\begin{array}{c}6.0875 \\
\times 10^{-5}\end{array}$ & $1.49 \%$ & $\begin{array}{c}5.7047 \\
\times 10^{-5}\end{array}$ & $\begin{array}{c}5.7110 \\
\times 10^{-5}\end{array}$ & $0.11 \%$ \\
\hline$\partial P_{f} / \partial \sigma_{R}$ & $\begin{array}{c}1.8002 \\
\times 10^{-4}\end{array}$ & $\begin{array}{c}1.6424 \\
\times 10^{-4}\end{array}$ & $8.76 \%$ & $\begin{array}{c}1.9905 \\
\times 10^{-4}\end{array}$ & $\begin{array}{c}1.8900 \\
\times 10^{-4}\end{array}$ & $5.05 \%$ & $\begin{array}{c}1.9402 \\
\times 10^{-4}\end{array}$ & $\begin{array}{c}1.8756 \\
\times 10^{-4}\end{array}$ & $3.32 \%$ \\
\hline$\partial P_{f} / \partial \sigma_{B}$ & $\begin{array}{c}1.3070 \\
\times 10^{-4}\end{array}$ & $\begin{array}{c}1.3149 \\
\times 10^{-4}\end{array}$ & $0.60 \%$ & $\begin{array}{c}1.4115 \\
\times 10^{-4}\end{array}$ & $\begin{array}{c}1.3927 \\
\times 10^{-4}\end{array}$ & $1.33 \%$ & $\begin{array}{c}9.5868 \\
\times 10^{-5}\end{array}$ & $\begin{array}{c}1.0501 \\
\times 10^{-4}\end{array}$ & $9.54 \%$ \\
\hline$\partial P_{f} / \partial \rho_{R B}$ & $\begin{array}{c}5.3670 \\
\times 10^{-5}\end{array}$ & $\begin{array}{c}5.4702 \\
\times 10^{-5}\end{array}$ & $1.92 \%$ & $\begin{array}{c}7.9386 \\
\times 10^{-5}\end{array}$ & $\begin{array}{c}7.8465 \\
\times 10^{-5}\end{array}$ & $1.42 \%$ & $\begin{array}{c}6.8337 \\
\times 10^{-5}\end{array}$ & $\begin{array}{c}6.7985 \\
\times 10^{-5}\end{array}$ & $0.52 \%$ \\
\hline$\partial P_{f} / \partial \sigma$ & $\begin{array}{c}2.2884 \\
\times 10^{-4}\end{array}$ & $\begin{array}{c}2.1739 \\
\times 10^{-4}\end{array}$ & $5.00 \%$ & $\begin{array}{c}2.5661 \\
\times 10^{-4}\end{array}$ & $\begin{array}{c}2.4754 \\
\times 10^{-4}\end{array}$ & $3.54 \%$ & $\begin{array}{c}2.2685 \\
\times 10^{-4}\end{array}$ & $\begin{array}{c}2.2545 \\
\times 10^{-4}\end{array}$ & $0.62 \%$ \\
\hline
\end{tabular}

\section{Conclusions}

1) When analyzing the reliability and its sensitivity of the structure, the paper adopts the mode of stratification and transforms the small failure probability into a series of continuous product of the large CFP, which enhances the efficiency of solving the problem. Moreover, the course isn't subject to the variable dimension, so it is suitable to answer the small failure probabilities in high dimensions.

2) Although the normal distribution is exampled to illustrate the algorithm in this paper, for the non-normal variables, only when the corresponding PDF is substituted into the function $f(x)$ instead of the variable-normalized transformation can they be solved. It is also adaptive to analyze the reliability of nonlinear implicit LSE.

3) In the paper, the reliability and its sensitivity are analyzed by directly sampling mode, by which can avoid the trifle resulting from the operation that the corrected variables are changed into independent ones. The examples show that the HMC which the SS is combined with direct Monte Carlo and IS approach together can highly simulate structural reliability and its sensitivity.

\section{Acknowledgements}

This work was financially supported by the Foundation of Hunan Provincial Natural Science of China(Grant No.2015JJ2015), the Foundation of Hunan Educational Department(Grant No.14C0094, 15C0122, 15C0123), the talent introduction foundation of Changsha University(Grant No.12004), Science and Technology Program of Changsha(Grant No.k1407020-11), and the Foundation of Hunan Province key built disciplines of China(2016). This support is gratefully acknowledged. We are also grateful to the referees whose comments were very helpful in the revision of this paper.

\section{References}

[1] Song, S. F., \& Lü, Z. Z. (2010). Reliability sensitivity analysis based on directional simulation for systems of multiple design points/failure modes with normal correlated variables. Acta Aeronautica et Astronautica Sinica, 31(1),109-118.

[2] Au, S. K., Cao, Z. J., \& Wang, Y. (2010). Implementing advanced Monte Carlo simulation under spreadsheet 
environment. Structural Safety, 32(3), 281-292.

[3] Au, S. K., \& Beck, J. L. (2001). Estimation of small failure probabilities in high dimensions by subset simulation. Probabilistic Engineering Mechanics, 16(4), 263-277.

[4] Frank, G. (2008). Adaptive radial-based importance sampling method for structural reliability. Structural Safety, 30(2), 533-542.

[5] Yan, X. K., Lü, Z. Z., \& Qiao, H. W. (2010). Conditional probability Markov chain simulation based reliability analysis method for nonnormal variables. Sci China Tech Sci, 53(8), 1434-1441.

[6] Au, S. K., Ching, J., \& Beck, J. L. (2007). Application of subset simulation methods to reliability benchmark problems. Structural Safety. 29(7), 183-193.

[7] Song, S. F., Lü, Z. Z., \& Qiao, H. W. (2009). Subset simulation for structural reliability sensitivity analysis. Reliability Engineering and System Safety, 94(3) ,658-665.

[8] Zhang, G. Q., \& Gong, X. S. (2012). Structural collaborative optimization based on hybrid GA-PSO algorithm with correlated variables. Journal of Mechanical Engineering, 48(15),113-125.

[9] Zio, E., \& Pedroni, N. (2010). An optimized line sampling method for the estimation of the failure probability of nuclear passive systems. Reliability Engineering and System Safety, 95(9),1300-1313.

[10] Li, H. S., \& Au, S. K. (2010). Design optimization using subset simulation algorithm. Structural Safety, 32(6), 384-392.

[11] Song, S. F., \& Lü, Z. Z. (2008). Reliability sensitivity analysis based on subset simulation and importance sampling. Chinese Journal of Theoretical and Applied Mechanics, 45(5),654-662.

[12] Ching, J., Beck, J. L., \& Au, S. K. (2005). Hybrid subset simulation method for reliability estimation of dynamical systems subject to stochastic excitation. Probabilistic Engineering Mechanics, 20(2), 199-214.

[13] Tan, M. L., \& Dong, J. L. (2010). Buckling analysis of thin rectangular plates under cosine-distributed compressive loads. Engineering Mechanics, 27(5) ,32-35.

[14] Rao, Z. G. (2003). Transmission Design of Planetary Gear. Beijing: Chemical Industry Press.

[15] Zhang, G .Q., Gong, X. S., \& Wang, H. H., et al. (2010). Multi-objective optimization design on gear train of planetary reducer in shield tunnelling machine based on reliably grey particle swarm optimization. Journal of Mechanical Engineering, 46(23),135-145.

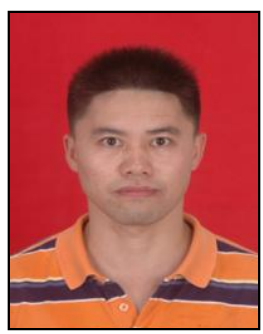

Ganqing Zhang is an associate professor of mechanical engineering field at Changsha University, China. He obtained his Ph.D from Chongqing University, China. He is a member of International Federation for the Theory of Machines and Mechanisms(IFToMM), and he is a peer reviewer for several journals. His areas of interest are reliability engineering, intelligence optimization, reliability-based optimization and uncertain analysis.

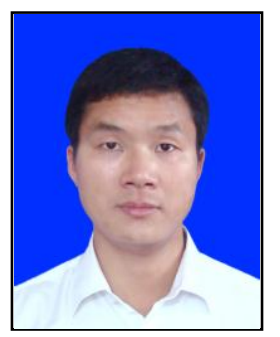

Yanghui Xiang is a lecturer of mechanical engineering field at Changsha University, China. He obtained his Master degree from Central South University, China. His areas of interest are reliability analysis and fault diagnosis. 


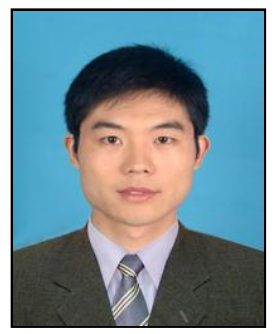

Lei Guo is a lecturer of mechanical engineering field at Changsha University, China. He obtained his Ph.D from China University of Petroleum-Beijing. His areas of interest are reliability analysis and optimization design.

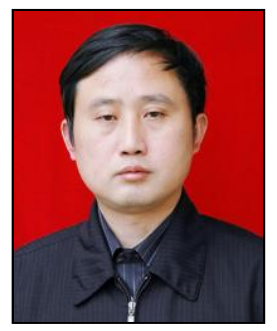

Huixin Guo is a full professor of mechanical engineering field at Changsha University, China. He obtained his master degree from Hunan University, China. and he is a peer reviewer for several journals. His areas of interest are reliability engineering, optimization design and uncertain analysis.

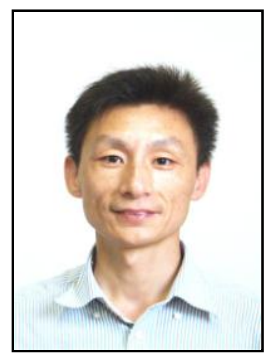

Yonghong Nie is an associate professor of mechanical engineering field at Changsha University, China. He obtained his Ph.D from Hunan University, China. His areas of interest are reliability analysis, fault diagnosis and noise control. 